\title{
Robust Control of Multi Degree of Freedom Robot Based on Disturbance Observer of Neural Network
}

\author{
Na Wang ${ }^{1}$, Jimeng Zhang ${ }^{1}, \mathrm{Ke} \mathrm{Xu}^{2,3^{*}}$ and Hu Junzhe ${ }^{4}$ \\ ${ }^{1}$ Henan Polytechnic Institute, Nanyang Henan 473000, China \\ ${ }^{2}$ Henan Institute of Technology, Xinxiang Henan 453000, China \\ ${ }^{3}$ Henan Key Equipment Engineering Research Center for New Energy Power Generation, Xinxiang Henan 453000, China \\ ${ }^{4}$ Kaiserslautern Technical University, Kaiserslautern Rhineland-Pfalz 67663, Germany
}

Received 1 February 2020; Accepted 12 April 2020

\begin{abstract}
A multi degree of freedom (DOF) robot is a complex and variable nonlinear system, and its control performance is affected by the inherent parameters of the model itself, friction, external disturbance, and other factors. A robust control method based on neural network disturbance observer was proposed in this study to improve the effect of time-varying system parameters and external disturbances on the control system performance. A new dynamic model of robot error was constructed by analyzing the characteristics of the robot system model. The total disturbance of the system was observed and compensated online on the basis of the neural network observer, and the effectiveness of the control method was verified through simulation. Results demonstrate that the robust adaptive control method with neural network disturbance observer reduces the maximum angular displacement error by 2.7 times and the maximum angular velocity tracking error by 2.14 times compared with the control method without observer when model parameter perturbation and external disturbance are found in the system. The maximum angular velocity error is 4 and 88.6 times lower than proportional derivative (PD) compensation control and traditional sliding mode control, respectively. The neural network disturbance observer can accurately track the total disturbance of the system. The input torque of the proposed control method has a small peak torque, which is $1 / 8$ and $1 / 2$ times lower than the sliding mode control and PD compensation control, respectively, and the control curve of the proposed control method is relatively smooth. The proposed method provides a reference for the multi DOF robot to achieve high-precision tracking in complex and changeable environment.
\end{abstract}

Keywords: Multi DOF robot, Neural network, Disturbance observer, Robust control, Sliding mode control

\section{Introduction}

A robot integrates advanced manufacturing technologies, such as precision, flexibility, intelligence, and software application development. Robots can achieve capacity increase, quality improvement, cost reduction, consumption reduction, and pollution reduction through the implementation of process detection, control, optimization, scheduling, management, and decision making. Therefore, robots are applied in automatic production, such as machining, automobile manufacturing, assembly, and logistics palletizing. The requirements of robot's dynamic performance, such as working efficiency, response velocity, track tracking accuracy, and stability time are continuously increasing. However, the robot is a complex multi timevarying and strong coupling dynamic system. Many uncertain factors, such as unmodeled dynamics, parameter measurement error, load change, external disturbance, and friction between joints, hinder the development of a precise dynamic model of the robot. These uncertain factors will seriously affect the control performance and dynamic quality of the system, especially for high-precision, highperformance, and high-speed robot system. Studying the control problem with strong suppression ability of uncertain interference, such as modeling error and external

*E-mail address: xk126sun@126.com

ISSN: 1791-2377 @ 2020 School of Science, IHU. All rights reserved.

doi:10.25103/iestr.133.16 disturbance, is particularly important.

Robots are developing toward multi degree of freedom (DOF), flexibility, two polarization (micro and giant), and direct drive. A multi DOF joint robot driven by traveling wave ultrasonic motor is suitable for high precision operation [1]. As medical intelligent auxiliary equipment, the new osteotomy robot can meet the requirements of the particularity of working object and the high precision of structural design [2]. In the case of high space and weight requirements, such as oil-immersed transformers, highprecision detection robot can realize precise observation at a specific point in the depth direction [3]. Flexible and adjustable high-precision abrasive belt grinding robot has become an effective means to improve blade surface integrity for meeting the requirements of precision machining of aeroengine blade surface [4]. The intelligent inspection robot of substation based on global satellite positioning technology can meet the practical needs of highprecision positioning, large-scale operation, and other projects in power inspection [5]. The developed heavy-duty friction stir welding (FSW) robot can realize smart welding ability under heavy-duty working condition in large working space and high-precision FSW of high-strength large-scale complex curved parts [6]. The 6-DOF industrial robot equipped with laser tracker linkage closed-loop control can complete high-precision assembly of satellite components [7]. However, the robot's model parameters and external disturbances are variable and uncertain in complex and changeable working environment. Corresponding control 
methods must be studied to achieve high-precision control.

This study proposes an error dynamic model with model parameter uncertainty and external disturbance in accordance with the structural characteristics of multi DOF robot, and sets $L_{2}$ gain antidisturbance index in accordance with robust control theory. A robust control method based on neural network and adaptive theory is presented to solve the influence of system time-varying parameters and disturbance on the system performance, thereby providing a scheme of high-precision tracking for robot servo system.

\section{State of the art}

Scholars have studied the tracking control of robots, mainly focusing on the adaptive control of system model parameter online estimation, sliding mode control of disturbance suppression, intelligent control, and other methods. Proportional integral derivative (PID) control was the most commonly used in industrial robots because it has the advantages of simple structure and easy physical realization. Huynh et al. [4] proposed a force/position hybrid control method of a double closed-loop PID controller for a 6-DOF parallel robot to ensure its safety and accuracy when contacting the object surface. The use of impedance control model ensured the safe and accurate interaction when contacting the object surface and eliminated the position error caused by the dynamic coupling effect between the mechanical arm and mechanical system vibration. The proposed method had high accuracy in the case of certain model and constant load, and the time-varying model parameters and load disturbance reduced the tracking performance of the robot. Zhang et al. [5] combined fuzzy control with PID control and designed a switch fuzzy PD control method that improved the dynamic performance of the system in terms of rise time, overshoot, and oscillation. However, the designed method adjusted the proportion and differential gain online through fuzzy rules in accordance with the angular displacement error and the rate of change signal of the robot joint, the structure of the controller was complex, and the fuzzy rules were difficult to determine. Internal model control had the advantages of simplicity, good tracking and adjusting performance, strong robustness, and elimination of unmeasurable disturbances. The flexibility of reducer leaded to the existence of resonance point in the joint servo control system of industrial robot, and the traditional PI control vibrated under the extreme position command. According to the elastic transmission model of single joint of industrial robot, Huang et al. [6] presented a vibration suppression method of industrial robot joint servo system based on the principle of internal model control, and the adopted method enhanced the robustness of the system by adding vibration suppression filter between position and velocity loops without additional sensors and complex control algorithm, which had more dependence than PID control method on model parameters. Liu [7] estimated the external disturbance online through a time delay observer to improve the dependence of internal model control on the model and to overcome the influence of external disturbance on the system performance. The introduction of time delay observer excited the highfrequency signal of the system that needed to be processed by low-pass filter, resulting in the complex structure of the control system. Meng et al. [8] introduced an adaptive mechanism into the internal model control, to eliminate the dependence of model parameters. However, the adaptive mechanism was sensitive to external disturbances and difficult to be used in high-precision and complex environments. In view of parameter uncertainty in the flexible joint robot system, Luc et al. [9] designed a robust adaptive control method based on cascade structure by using full state feedback controller, integral term as internal control loop, and calculated torque as external control loop. The adaptive chattering tracking was realized by adaptive friction compensation, the adaptive tracking technique ensured the trajectory tracking of the robot, but the premise was that the robot model structure was completely known and linear under unknown parameters, and its joint velocity was also available. The proposed method was frequently used in combination with sliding mode control, disturbance observer, and intelligent control. The time delay observer realized the real-time estimation of external disturbance, and Baek et al. [10] introduced the time delay signal to cancel the complex nonlinear dynamics and disturbance of the robot. During operation, the control gain was automatically adjusted in real time, thereby improving the influence of parameter uncertainty and external disturbance on the system performance at the same time. However, the proposed method needed low-pass filtering in time delay estimation, thereby increasing the structure complexity of the controller. The advantage of robust technology was that prior information of robot model parameters/structure or disturbance, such as sliding mode control, wasn't needed in tracking. Rubio et al. [11] divided the robot model into two parts, namely, deterministic and total disturbance parts. In the deterministic part, the nonlinear feedback control was employed to compensate the uncertainties and external disturbances of the model using the switch robust control with strong robustness. However, the adopted method required the model parameters to meet the matching conditions, and its switch control had chattering phenomenon, thereby limiting the application of the system. Baek et al. [12] combined adaptive control method and sliding mode control to improve the chattering problem of sliding mode control, and the designed adaptive sliding mode control method ensured that the derivative of adaptive gain was inversely proportional to the sliding variable in any small neighborhood of the sliding manifold. The proposed method provided remarkable ability of fast adaption and chattering suppression near the sliding manifold, but it didn't eliminate the influence of chattering on system performance. Nair et al. [13] developed an adaptive terminal sliding mode control method that completely utilized the strong ability of terminal attractor to suppress the system interference, and adjusted the control gain online with adaptive control. The designed method ensured that the system tracks the expected instructions in a limited time under various disturbances, but a fractional calculus operator was used in the control, and its construction was complex in practice and had limited application. The predictive control model predicted the future dynamic model, control effect of on-line repeatedly optimization calculation and rolling implementation and feedback correction of model error, to effectively overcome the uncertainty, nonlinearity, and parallelism of the process. Worthmann team [14] and Chen team [15] used the designed method in nonholonomic mobile robot systems. The former didn't consider the system uncertainty, whereas the latter considered the effects of input constraints and unmodeled dynamics. Although the method was robust to uncertainty, it was sensitive to the changes of system parameters based on the model parameters of the system. Incremona et al. [16] combined adaptive terminal sliding mode control with 
sliding mode control to overcome the influence of sensitivity in the change of system parameters, but the adopted method increased the complexity of the system controller and made it difficult to determine the control parameters. Liu et al. [17] used a backstep method in the control of robot hand grasping, but the backstep control needed differential derivation in each step that caused knowledge explosion and other problems. Peng et al. [18] applied sliding mode and adaptive theories to backstepping control, and the proposed method had strong robustness to system parameter timevarying and disturbance, but its controller structure was relatively complex and the introduction of adaptive mechanism reduced the dynamic performance of the system. Fuzzy system and neural network had high nonlinear approximation ability and were applied as a powerful tool to solve complex nonlinear systems. Scholars had applied fuzzy control, neural network control and so on to the robot, and introduced an intelligent control method of the robot [19-21]. In the application, the structures of neural network control and fuzzy control were more complex, the weight and structure of the network were difficult to determine, and the rules of fuzzy control had no unified form so far. Thus, several difficulties were encountered in the application.

Most of the above studies focused on the influence of system model parameter uncertainty, and had little influence on the friction torque, load disturbance and model parameter uncertainty of the system. This study proposes an error dynamic model of $L_{2}$ gain antijamming based on the characteristics of an uncertain robot model. The total disturbance of the system is observed and compensated using a neural network observer, and a high-performance robust control method is designed to provide a basis for the application of high-precision tracking of robot in complex and changeable environment.

The remainder of this study is organized as follows. Section 3 designs the dynamic model of uncertain multi DOF robot, proposes a robust adaptive control method based on neural network observation compensation, and conducts stability analysis. Section 4 verifies that the proposed control method is more effective than the traditional control method. Section 5 summarizes the conclusions.

\section{Methodology}

\subsection{System description}

A joint robot, also called joint arm robot or joint manipulator, is a common form of industrial robot in today's industrial field and is suitable for mechanical automation in many industrial fields. Fig. 1 shows a typical multi joint robot, and Fig. 2 presents the structure and dimensions of a dual-joint robot.

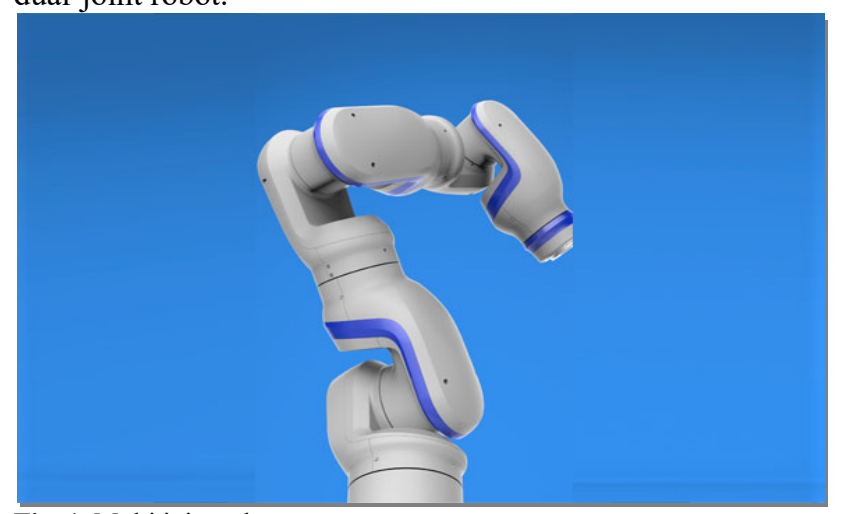

Fig. 1. Multi joint robot

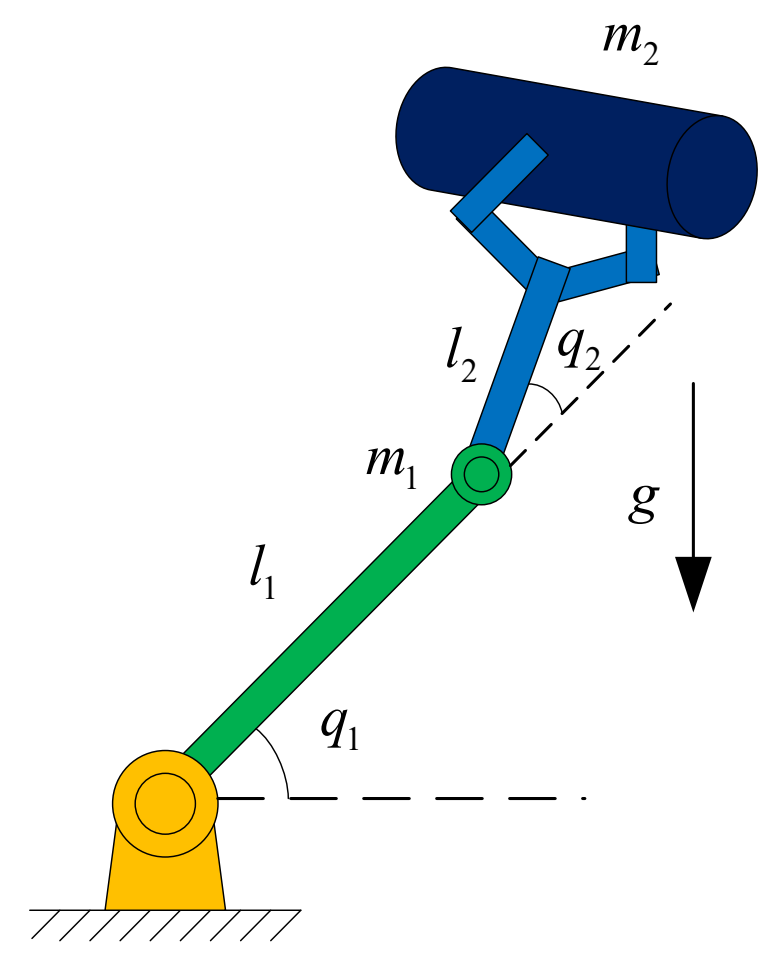

Fig. 2. Structure diagram of double-joint robot

The controlled object is an $n$-joint multi DOF robot, and its dynamic model is expressed as follows [9]:

$$
M_{0}(q) \ddot{q}+G_{0}(q, \dot{q}) \dot{q}+V_{0}(q)+\Delta(q, \dot{q})+d=\tau
$$

where $q \in R^{n}, \quad \dot{q} \in R^{n}$, and $\ddot{q} \in R^{n}$ are the angular displacement, angular velocity, and angular acceleration vectors of the robot, respectively. $M_{0}(q) \in R^{n \times n}$ is the nominal inertia matrix vector of the robot (symmetric positive definite). $G_{0}(q, \dot{q}) \in R^{n}$ is the composite matrix vector of centrifugal and Coriolis forces. $V_{0}(q) \in R^{n}$ is the nominal-scale gravity matrix vector. $\tau \in R^{n}$ is the control torque vector of the actuator. $d \in R^{n}$ is the total disturbance of robot system composed of external disturbance, friction moment, and unmodeled dynamics. $\Delta(q, \dot{q})$ is the uncertainty caused by system model parameters.

The dynamic model of robot system Eq. (1) satisfies the following characteristics:

(1) Characteristic 1: $M_{0}(q)-2 G_{0}(q, \dot{q})$ is a skew symmetric matrix, that is, $q^{T}\left(M_{0}(q)-2 G_{0}(q, \dot{q})\right) q=0$.

(2) Characteristic 2: Inertia matrix $M_{0}(q)$ is a symmetric positive definite matrix with a positive number that satisfies the inequality as follows: $m_{1}\|q\|^{2} \leq q^{T} M_{0}(q) q \leq m_{2}\|q\|^{2}$.

(3) Characteristic 3: A parameter vector that depends on the parameters of the manipulator is found, making $M_{0}(q)$, $G_{0}(q, \dot{q}), V_{0}(q)$, and $d$ satisfy the linear relationship as follows: $\quad M_{0}(q) \vartheta+G_{0}(q, \dot{q}) \rho+V_{0}(q)+d=\Phi\left(q, q^{\prime}, \rho, \vartheta\right) P$, where $\Phi\left(q, q^{\prime}, \rho, \vartheta\right)$ is the regression matrix of the known joint variable function, that is, the known function matrix of the generalized coordinates of the robot and its derivatives of 
each order. $P$ is the unknown constant parameter vector that describes the quality characteristics of the robot. $\vartheta$ and $\rho$ are the corresponding position and velocity vector, respectively.

(4) Control objective: Considering parameter perturbation $\Delta(q, \dot{q})$ and external disturbance in the system, control torque $\tau$ is designed to realize the precise tracking of the robot's position and velocity.

\subsection{Controller design and stability analysis}

Assuming that the desired trajectory of the robot is $q_{r}$ and the tracking error of system is $e=q-q_{r}$, the feedforward control law is:

$\tau_{1}=\tau-M_{0}(q) \ddot{q}+G_{0}(q, \dot{q}) \dot{q}+V_{0}(q)$

Substituting feedforward control law $\tau_{1}$ into Eq. (1), the system model can be expressed as:

$M_{0}(q) \ddot{e}+G_{0}(q, \dot{q}) \dot{e}+\Delta=\tau_{1}$

where $\Delta=\Delta(q, \dot{q})+d$ is the total disturbance of the system, $\Delta$ is estimated and compensated online using radial basis function neural network and is expressed as follows:

$\Delta=W_{d}^{*} \varphi_{d}+\varepsilon_{d}$

where $\varphi_{d}$ is the neuron excitation function of the radial basis function neural network, $W_{d}^{*}$ is the weight of the ideal neural network, and $\varepsilon_{d}$ is the approximation error. When approximation error $\varepsilon_{d}$ of the neural network is regarded as the external disturbance of the system and an evaluation index signal (sliding surface function) $z$ is defined, then gain $L_{2}$ of the system is $J=\sup _{\left|\varepsilon_{d}\right| \neq 0} \frac{\|z\|_{2}}{\left\|\varepsilon_{d}\right\|_{2}}$.

The following expressions can be obtained by substituting $\Delta$ into Eq. (3):

$M_{0}(q) \ddot{e}+G_{0}(q, \dot{q}) \dot{e}+W_{d}^{*} \varphi_{d}+\varepsilon_{d}=\tau_{1}$ follows:

The designed sliding mode function is expressed as

$s=\dot{e}+c \dot{e}$

where $c>0$, and Eq. (6) satisfies the Hurwitz condition.

Defining new error state variables $\theta_{1}=e$ and $\theta_{2}=s$, then the expressions can be obtained as follows:

$\left\{\begin{array}{l}\dot{\theta}_{1}=\theta_{2}-c \theta_{1} \\ M_{0} \dot{\theta}_{2}=-G_{0} \theta_{2}+M_{0} c \dot{e}+G_{0} c e-W_{d}^{*} \varphi_{d}-\varepsilon_{d}+\tau_{1}\end{array}\right.$

Theorem When adaptive control law Eq. (8) and adaptive law Eq. (9) are adopted for Eq. (7) composed of new error state variable and the evaluation function is taken $z=s$, the closed-loop system satisfies $J \leq \lambda$, where $\lambda>0$ is a constant.
$\tau_{1}=-M_{0} c \dot{e}-G_{0} c e-\frac{1}{2 \lambda^{2}} \theta_{2}+\hat{W}_{d} \varphi_{d}-\frac{1}{2} \theta_{2}$

$\dot{\hat{W}}_{d}=-\beta \theta_{2} \varphi_{d}^{T}$

where $\hat{W}_{d}$ is the estimated value of neural network weight $W_{d}$, and its error is $\tilde{W}_{d}=\hat{W}_{d}-W_{d}^{*} . \beta$ is the adaptive updating coefficient of weight.

Proof. Lyapunov function is defined as $V=\frac{1}{2} \theta_{2}^{T} M_{0} \theta_{2}+\frac{1}{2 \beta} \operatorname{tr}\left(\tilde{W}_{d}^{T} \tilde{W}_{d}\right)$, where $\operatorname{tr}(*)$ is expressed as matrix trace $*$.

The derivation of Lyapunov function along error dynamic Eq. (7) is expressed as follows:

$$
\begin{aligned}
\dot{V} & =\theta_{2}^{T} M_{0} \dot{\theta}_{2}+\frac{1}{2} \theta_{2}^{T} \dot{M}_{0} \theta_{2}+\frac{1}{\beta} \operatorname{tr}\left(\dot{\tilde{W}}_{d}^{T} \tilde{W}_{d}\right) \\
= & \theta_{2}^{T}\left(-G_{0} \theta_{2}+M_{0} c \dot{e}+G_{0} c e-W_{d}^{*} \varphi_{d}-\varepsilon_{d}+\tau_{1}\right) \\
& +\frac{1}{2} \theta_{2}^{T} \dot{M}_{0} \theta_{2}+\frac{1}{\beta} \operatorname{tr}\left(\dot{\tilde{W}}_{d}^{T} \tilde{W}_{d}\right)=\theta_{2}^{T}\left(-G_{0} \theta_{2}-W_{d}^{*} \varphi_{d}-\varepsilon_{d}-\right. \\
& \left.\frac{1}{2 \lambda^{2}} \theta_{2}+\hat{W}_{d} \varphi_{d}-\frac{1}{2} \theta_{2}\right)+\frac{1}{2} \theta_{2}^{T} \dot{M}_{0} \theta_{2}+\frac{1}{\beta} \operatorname{tr}\left(\dot{\tilde{W}}_{d}^{T} \tilde{W}_{d}\right) \\
= & \theta_{2}^{T}\left(-\varepsilon_{d}-\frac{1}{2 \lambda^{2}} \theta_{2}+\tilde{W}_{d} \varphi_{d}-\frac{1}{2} \theta_{2}\right)+\frac{1}{2} \theta_{2}^{T}\left(\dot{M}_{0}-2 G_{0}\right) \theta_{2} \\
& +\frac{1}{\beta} \operatorname{tr}\left(\dot{\tilde{W}}_{d}^{T} \tilde{W}_{d}\right)=-\theta_{2}^{T} \varepsilon_{d}-\frac{1}{2 \lambda^{2}} \theta_{2}^{T} \theta_{2}+\theta_{2}^{T} \tilde{W}_{d} \varphi_{d}-\frac{1}{2} \theta_{2}^{T} \theta_{2} \\
& +\frac{1}{\beta} \operatorname{tr}\left(\dot{\tilde{W}}_{d}^{T} \tilde{W}_{d}\right)
\end{aligned}
$$

The expressions are defined as follows:

$F=\dot{V}-\frac{1}{2} \beta^{2}\left\|\varepsilon_{d}\right\|^{2}+\frac{1}{2}\|z\|^{2}$

Furthermore,

$$
\begin{aligned}
F= & -\theta_{2}^{T} \varepsilon_{d}-\frac{1}{2 \lambda^{2}} \theta_{2}^{T} \theta_{2}+\theta_{2}^{T} \tilde{W}_{d} \varphi_{d}-\frac{1}{2} \theta_{2}^{T} \theta_{2} \\
& +\frac{1}{\beta} \operatorname{tr}\left(\dot{\tilde{W}}_{d}^{T} \tilde{W}_{d}\right)-\frac{1}{2} \beta^{2}\left\|\varepsilon_{d}\right\|^{2}+\frac{1}{2}\|z\|^{2}
\end{aligned}
$$

where $\quad-\theta_{2}^{T} \varepsilon_{d}-\frac{1}{2 \lambda^{2}} \theta_{2}^{T} \theta_{2}-\frac{1}{2} \beta^{2}\left\|\varepsilon_{d}\right\|^{2}=-\frac{1}{2}\left\|\frac{1}{\lambda} \theta_{2}+\lambda \varepsilon_{d}\right\|^{2} \leq 0$, $\theta_{2}^{T} \tilde{W}_{d} \varphi_{d}+\frac{1}{\beta} \operatorname{tr}\left(\dot{\tilde{W}}_{d}^{T} \tilde{W}_{d}\right)=0$, and $-\frac{1}{2} \theta_{2}^{T} \theta_{2}+\frac{1}{2}\|z\|^{2}=0$, thus $F \leq 0$. In accordance with the definition of function $F$, there is $\dot{V} \leq \frac{1}{2} \beta^{2}\left\|\varepsilon_{d}\right\|^{2}-\frac{1}{2}\|z\|^{2}$. The Hamilton Jacobi Inequality theorem states that angular displacement error $e$ and angular velocity error $\dot{e}$ of the robot meet the $L_{2}$ gain robust convergence requirements when $L_{2}$ 's gain $J$ of closed-loop system Eq. (7) is less than or equal to given value $\lambda$.

\section{Results analysis and discussion}

Taking the dual-joint multi DOF robot as an example, the effectiveness of the proposed method is verified through online interference estimation and compensation method of neural network. The important model parameters of the manipulator are expressed as follows:

$$
M_{0}(q)=\left[\begin{array}{cc}
1+2.4 \cos q_{2} & 1+1.2 \sin x_{2} \\
1+1.2 \sin x_{2} & 1
\end{array}\right]
$$


$G_{0}(q)=\left[\begin{array}{cc}-1.5 \dot{q}_{2} \sin q_{2} & -1.5\left(\dot{q}_{1}+\dot{q}_{2}\right) \sin q_{2} \\ -1.5 q_{1} \sin q_{2} & 0\end{array}\right]^{T}$

$V_{0}(q)=\left[\begin{array}{c}2.5 \cos q_{2}+1.2 \cos \left(q_{1}+q_{2}\right) \\ 1.2 \cos \left(q_{1}+q_{2}\right)\end{array}\right]^{T}$

$d=\left[\begin{array}{l}35 \operatorname{sgn} q_{2} \\ 35 \operatorname{sgn} q_{4}\end{array}\right]^{T}$

The desired command is set as $q_{1 d}=q_{2 d}=\sin t$, and the initial positions are all zero. The parameters of the sliding surface is $c=25$, the updating coefficient of the neural network is $\beta=1600$, the $L_{2}$ gain given index is $\lambda=0.05$, and the parameters of the radial basis function neural network are $c_{i}=(-0.9,-0.6,-0.3,0,0.3,0.6,0.9) \quad$ and $b_{i}=20$. The proposed method is compared with PD compensation control, traditional sliding mode control, and the proposed method without disturbance observer to verify its effectiveness.

\subsection{Tracking performance comparison of the system} without interference

Fig. 3, 4 and 5 show the position tracking error, velocity tracking error, and control input curve of PD compensation control, traditional sliding mode control, and the proposed method.

Fig. 3 shows that the three methods exhibit extremely good performance in achieving position tracking without external disturbance. In the initial stage, PD compensation control has a large position overshoot and is approximately $8 \times 10^{-2} \mathrm{rad}$, sliding mode control is approximately $2 \times 10^{-2} \mathrm{rad}$, and the proposed method is approximately $1 \times 10^{-2} \mathrm{rad}$. The accuracy of the proposed method is 8 and 2 times higher than PD compensation control and traditional sliding mode control, respectively, especially the position tracking accuracy of joint 2 can reach $4 \times 10^{-5} \mathrm{rad}$, which is extremely suitable for high-precision tracking operations.
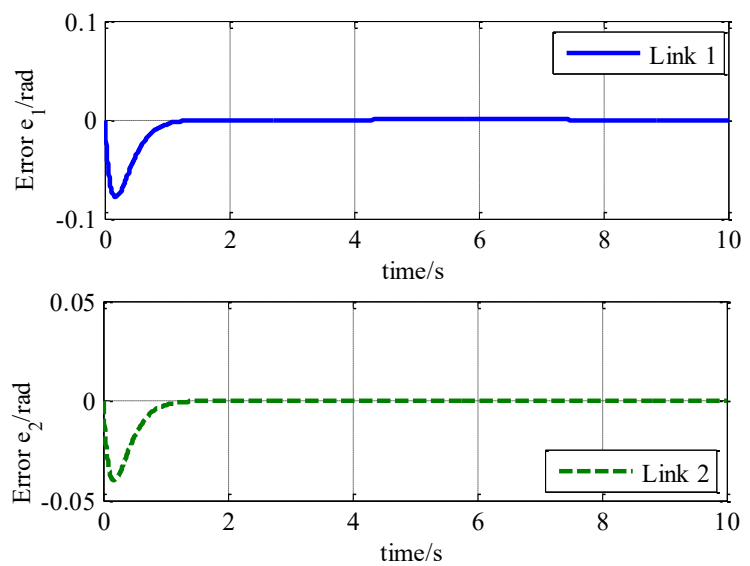

(a) Joint position tracking error of PD control
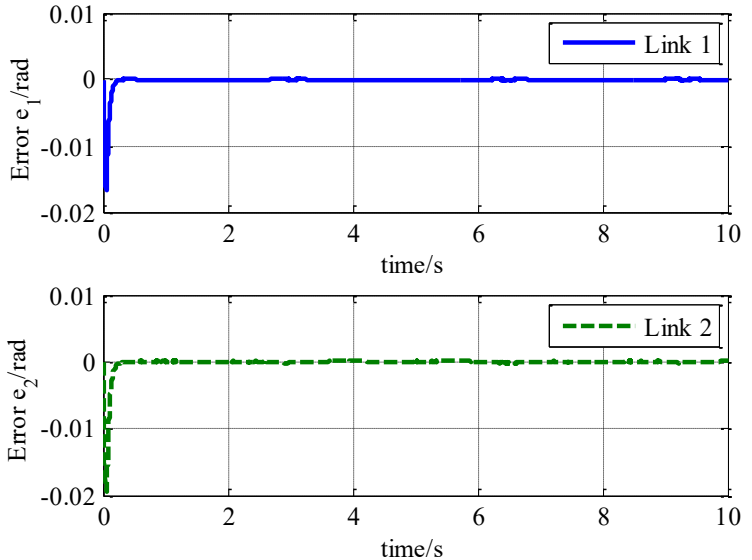

(b) Joint position tracking error of sliding mode control
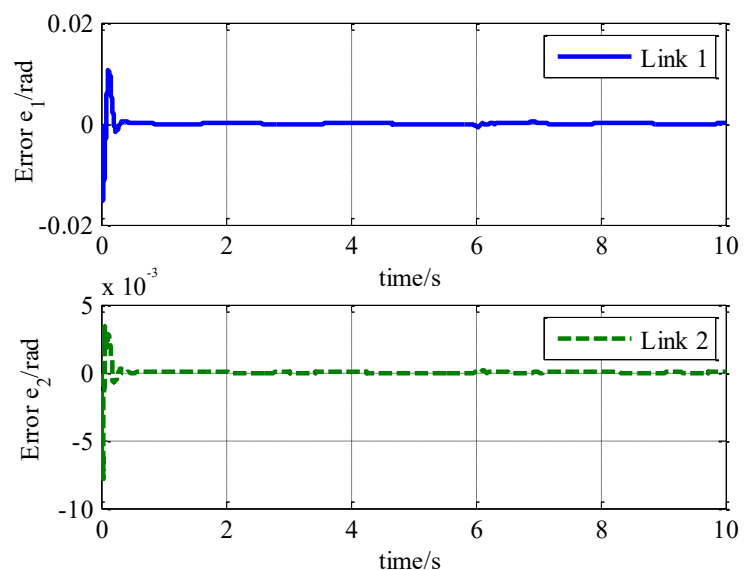

(c) Joint position tracking error of the proposed method

Fig. 3. Robot position tracking error without interference
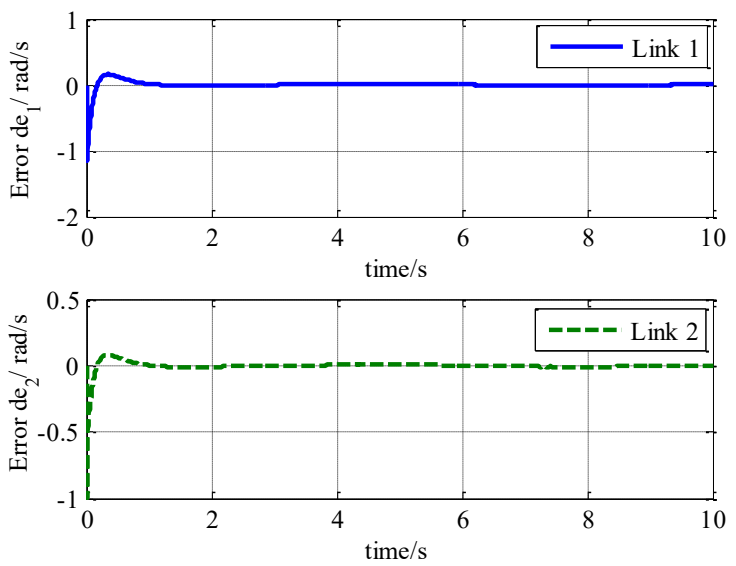

(a) Joint velocity tracking error of PD control
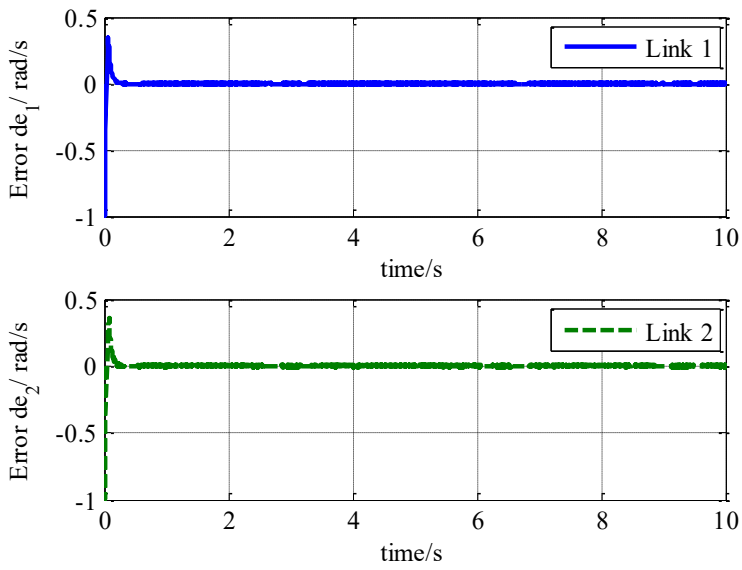

(b) Joint velocity tracking error of sliding mode control 

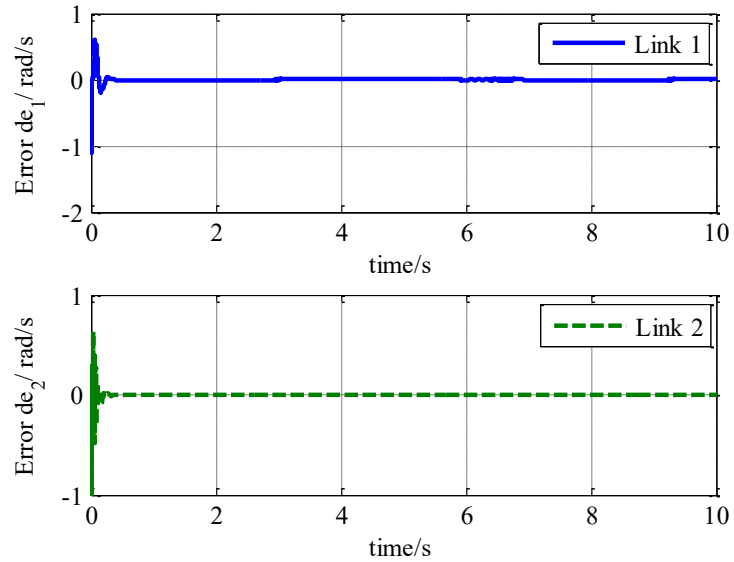

(c) Joint velocity tracking error of the proposed method Fig. 4. Robot velocity tracking error without interference
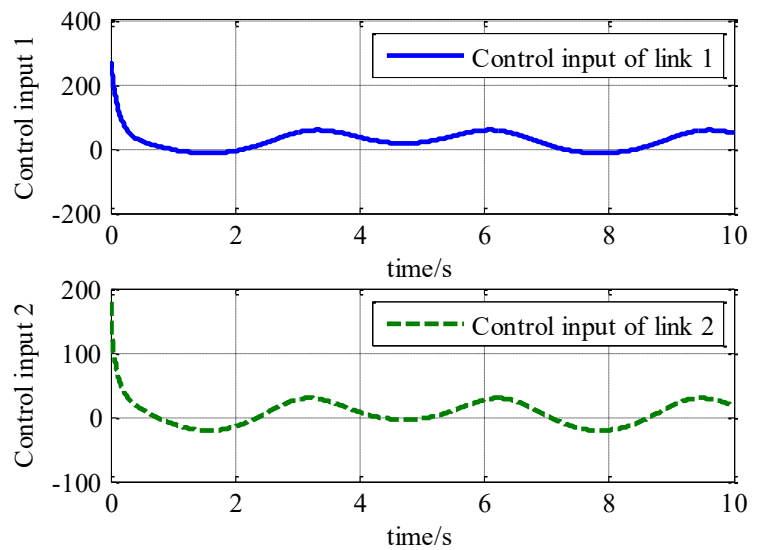

(a) Control input curve of PD control
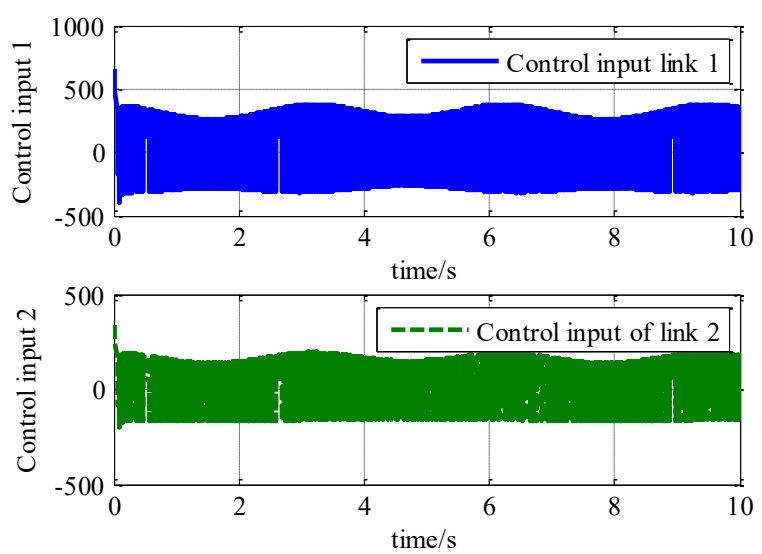

(b) Control input curve of sliding mode control
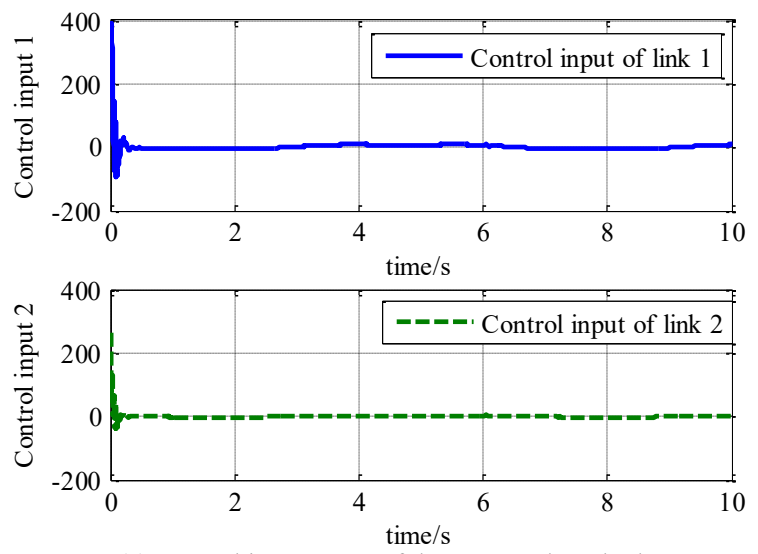

(c) Control input curve of the proposed method

Fig. 5. Control input curve without interference

\subsection{Tracking performance comparison of the system with} interference

Fig. 6, 7 and 8 show the position tracking error, velocity tracking error, and control input curve of PD compensation control, traditional sliding mode control, and the proposed method with friction moment disturbance, respectively.
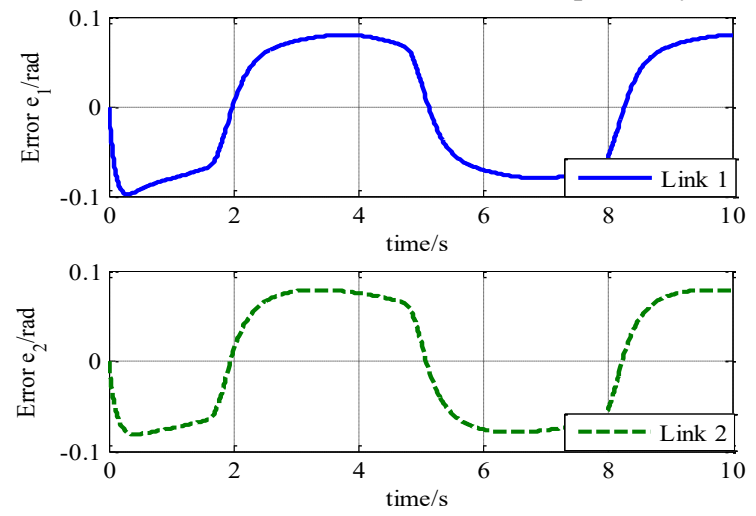

(a) Joint position tracking error of PD compensation control
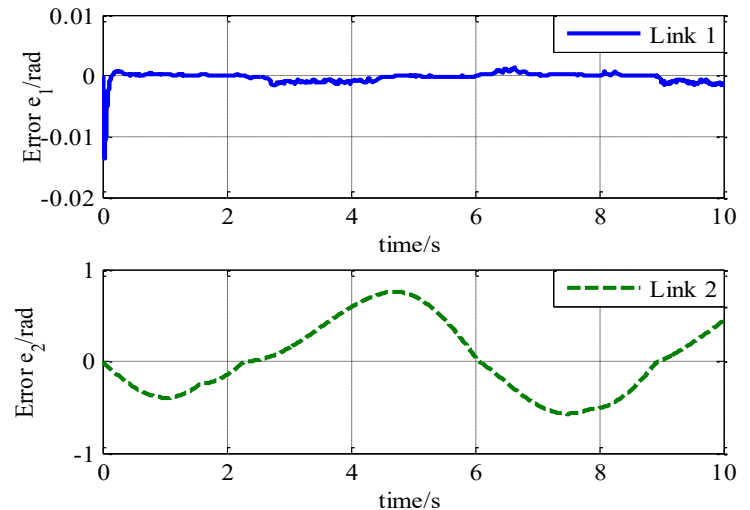

(b) Joint position tracking error of sliding mode control
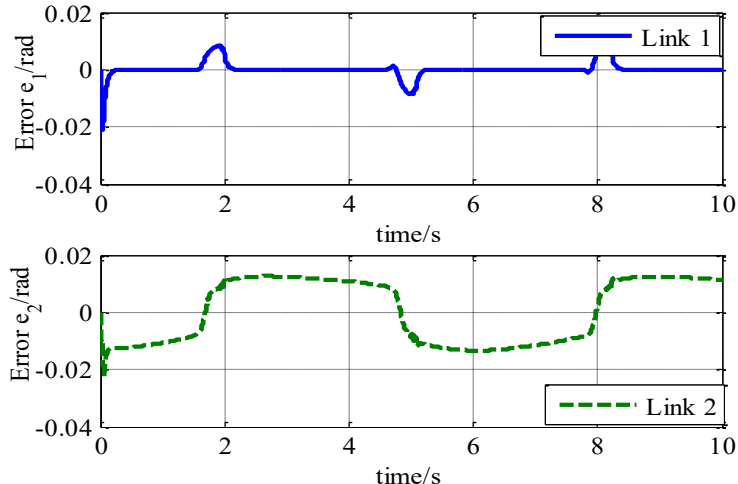

(c) Joint position tracking error of robust control without disturbance compensation
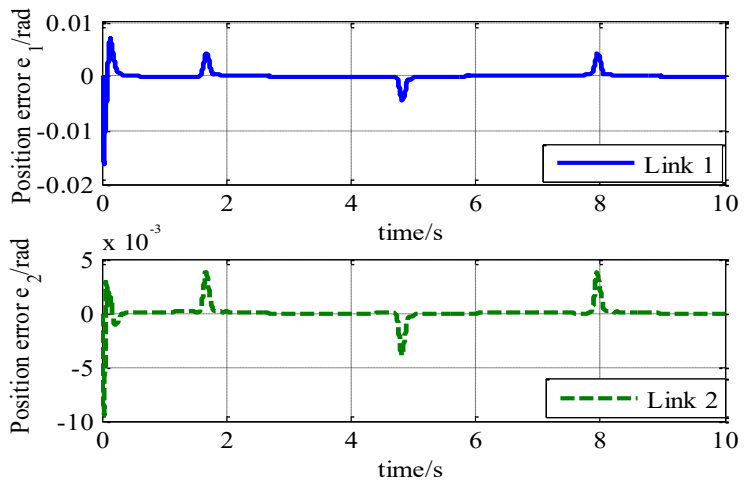

(d) Position tracking error of robust control with neural network disturbance compensation

Fig. 6. Robot position tracking error with interference 
Fig. 6 shows the position tracking error curve with friction moment disturbance. The conclusions can be summarized as follows: (1) The position tracking error of PD compensation control reaches $8 \times 10^{-2} \mathrm{rad}$ and is consistent with the change trend of disturbance. (2) The position tracking error of sliding mode control joint 2 reaches $0.76 \mathrm{rad}$. (3) The position tracking error of robust control without compensation is $0.16 \mathrm{rad}$. (4) The joint 1 tracking error compensated by neural network is $6 \times 10^{-2} \mathrm{rad}$, and the accuracy of joint 2 is within $5 \times 10^{-3} \mathrm{rad}$.
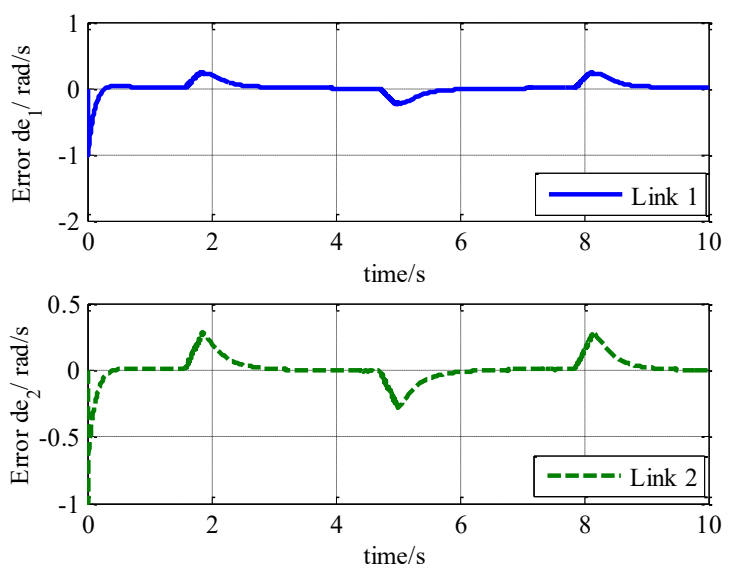

(a) Joint velocity tracking error curve of PD control
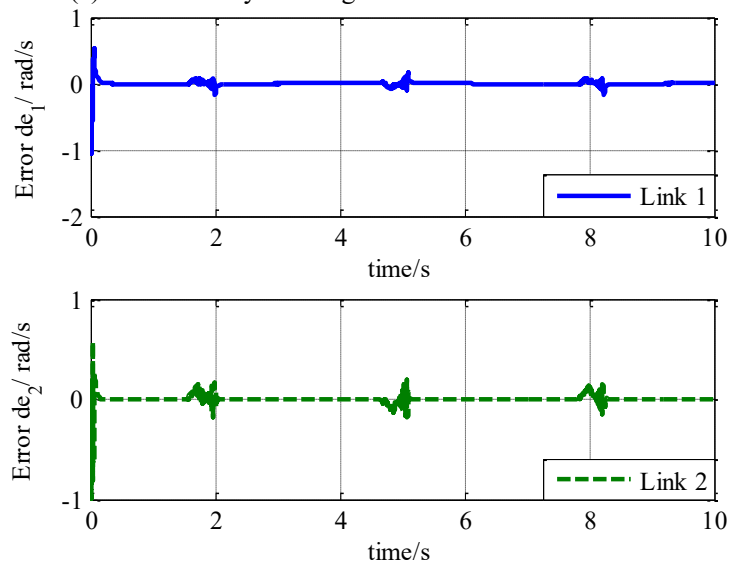

(b) Joint velocity tracking error curve of sliding mode control
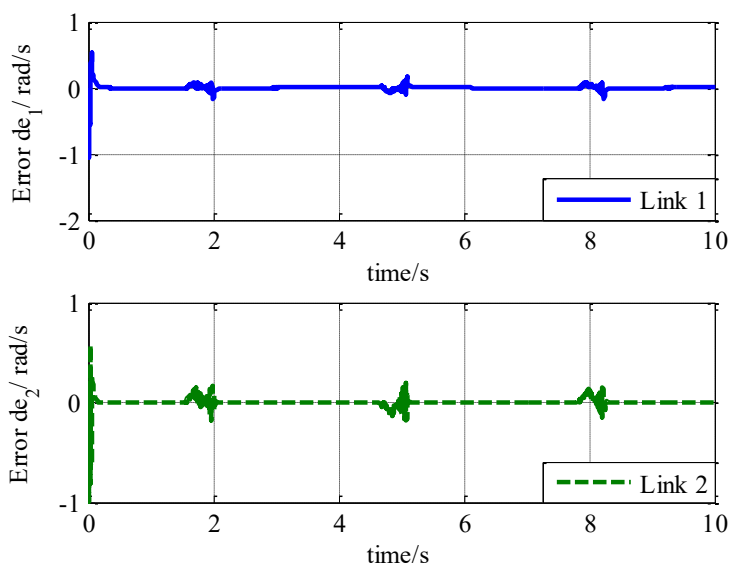

(c) Joint velocity tracking error curve of robust control without disturbance compensation
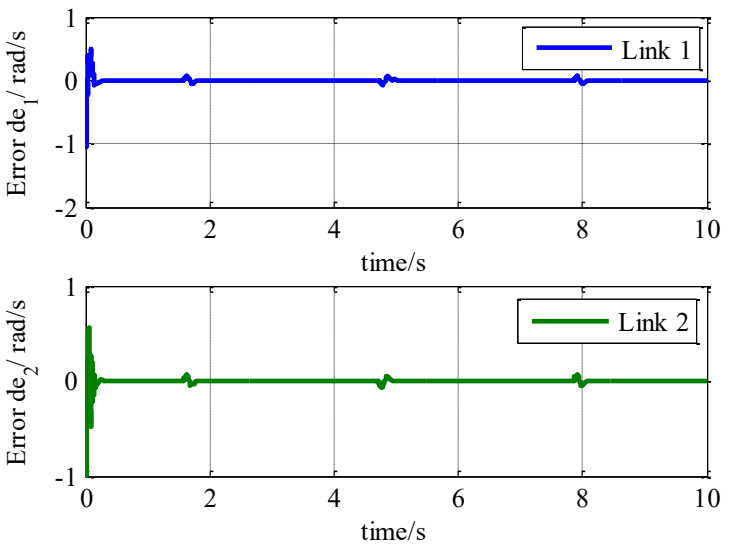

(d) Joint velocity error curve of robust control with neural network disturbance compensation

Fig. 7. Robot velocity tracking error with interference

Fig. 7 shows the tracking error curve of joint velocity. The conclusions can be summarized as follows: (1) The existence of uncertain friction torque increases the velocity tracking error of PD control and sliding mode control, the maximum velocity error of PD control reaches $0.0285 \mathrm{rad} / \mathrm{s}$, and sliding mode control is $0.62 \mathrm{rad} / \mathrm{s}$. (2) The maximum velocity error of robust control without compensation is $0.0175 \mathrm{rad} / \mathrm{s}$. (3) The maximum velocity tracking error of the proposed method is $0.007 \mathrm{rad} / \mathrm{s}$, and its tracking accuracy are 4, 88.6, and 2.5 times higher than PD compensation control, sliding mode control, and robust control without compensation, respectively, which can well realize the tracking of robot joint velocity.
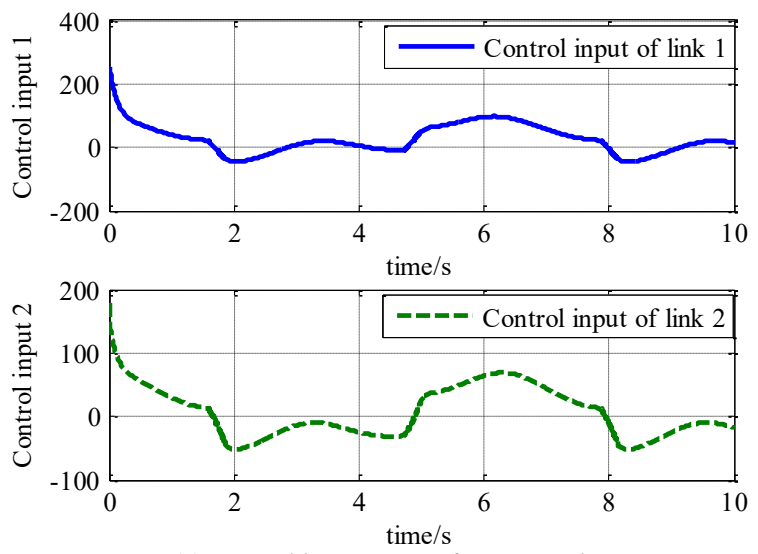

(a) Control input curve of PD control
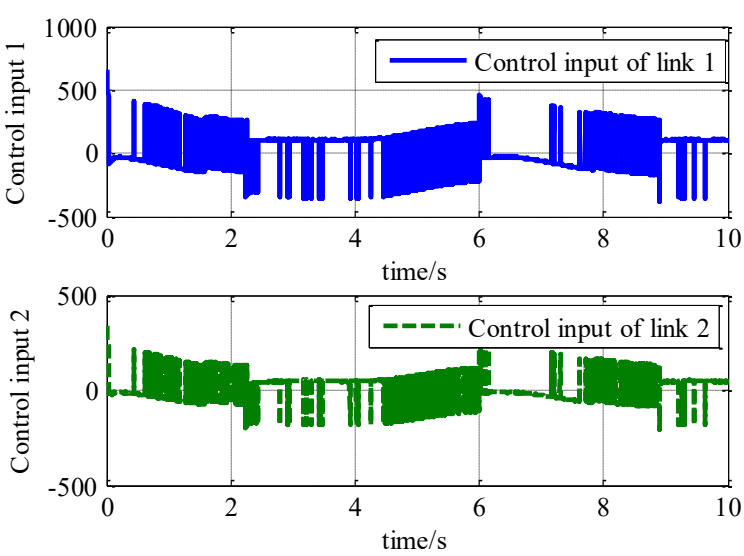

(b) Control input curve of sliding mode control 

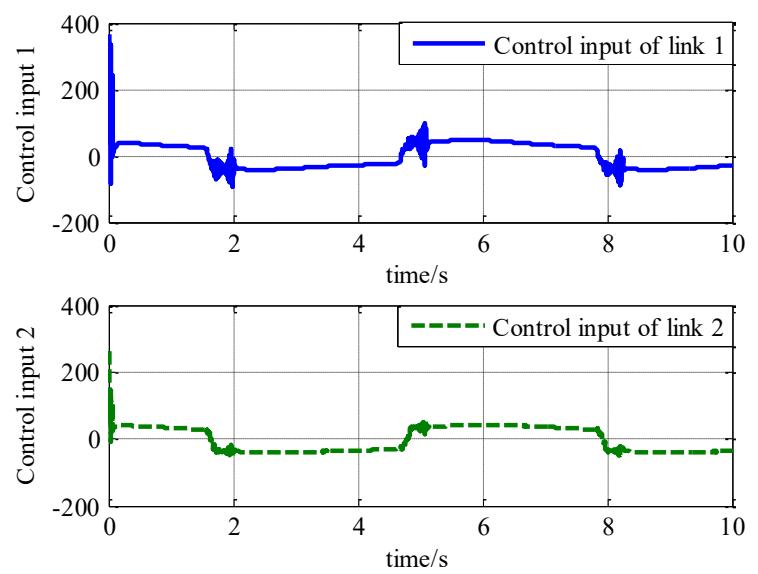

(c) Control input curve of robust control without disturbance
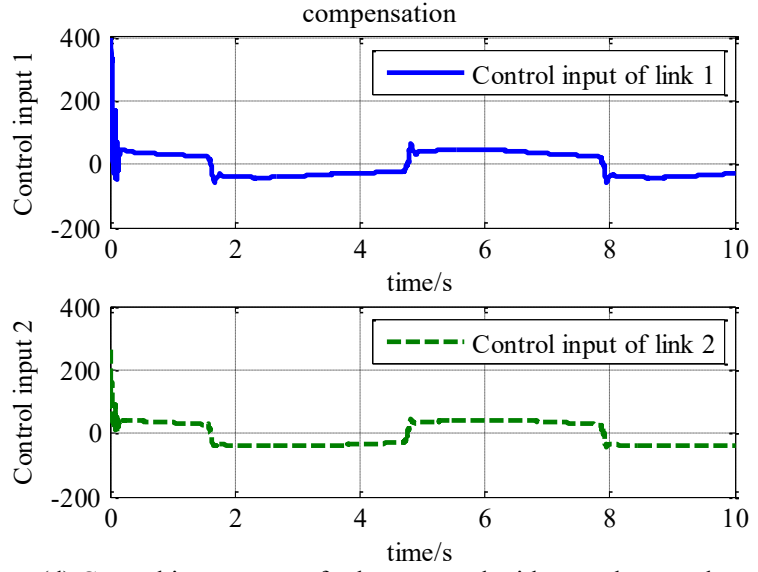

(d) Control input curve of robust control with neural network disturbance compensation

Fig. 8. Control input curve of the system with interference

Fig. 8 shows the control input curve of the system with interference. The conclusions can be summarized as follows: (1) PD compensation control and sliding mode control have large control inputs, the input of PD compensation control reaches $200 \mathrm{~nm}$, and the input of sliding mode control reaches $500 \mathrm{~nm}$. (2) The input of robust control without compensation is reduced to approximately $350 \mathrm{~nm}$, and a large fluctuation is found in the velocity conversion. (3) The input curve of the proposed control method is relatively smooth, no overshoot is found in the velocity conversion, and the steady-state control input of the proposed control method is $1 / 2$ and $1 / 8$ smaller than PD compensation control and sliding mode control inputs, respectively. Thus, the proposed method has better dynamic performance.
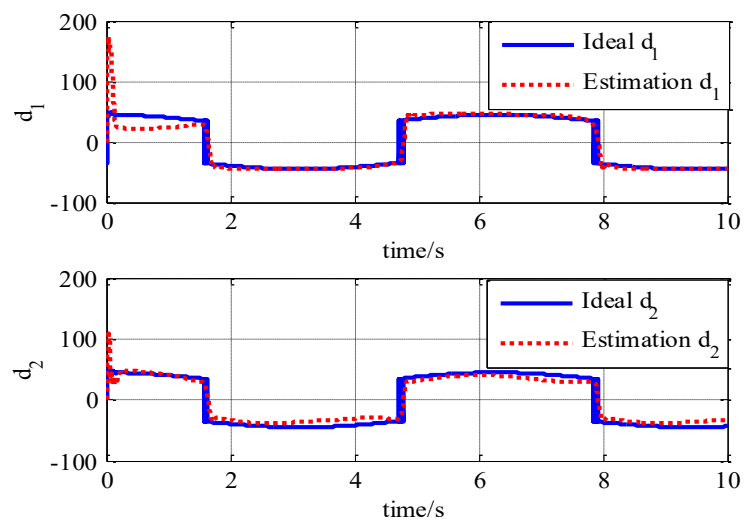

Fig. 9. Online estimation of uncertainty
Fig. 9 shows that the uncertainty of the system is estimated online using neural network, and the designed neural network observer can better track the corresponding disturbance of two joints.

\section{Conclusions}

The characteristics of system uncertainty was analyzed on the basis of the structural characteristics of the robot to overcome the influence of parameter perturbation and external interference on the robot joint tracking accuracy in the multi DOF robot system. The system performance was studied on the basis of neural network and adaptive control theory. The conclusions can be summarized as follows:

(1) The designed disturbance observer based on neural network can realize the real-time estimation of uncertainty and external disturbance caused by time-varying parameters in the system model.

(2) The proposed robust control method with neural network estimation compensation can improve the position and velocity tracking accuracy of robot joints and is 2.7 and 2.14 times higher than the control method without compensation, respectively. The maximum error of angular velocity is reduced by 4 and 88.6 times compared with PD compensation control and sliding mode control, respectively.

(3) The input curve of robust control with neural network disturbance compensation is smoother than PD compensation control and traditional sliding mode control, and its control energy is smaller.

Thus, simulation experiment and theory are combined to improve the effect of uncertain factors, such as model parameters and external disturbance of the multi DOF robot system. The proposed control method has simple structure, convenient for physical realization, and suitable for the actual working conditions, which can serve as guide for the subsequent application in the seam tracking of highperformance robots.

\section{Acknowledgements}

This work was supported by the Key Scientific Study Projects of Higher Education Institutions of Henan Province (Grant Nos. 20B470003 and 18B470007) and the Promotion Special Project of Scientific Study Program of Henan Province (Grant Nos. 202102210298, 202102210134 and 202102210084).

This is an Open Access article distributed under the terms of the Creative Commons Attribution License

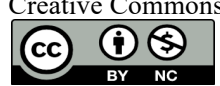




\section{References}

1. Hua, L., Feng, S., Gu, J. P., Zhang, J. S., "Precision positioning control of joint with multi-degrees of freedom based on travelingwave ultrasonic motor". Manufacturing Automation, 30(10), 2008, pp.89-93.

2. Gong, J. L, Li, X., Li, M., Zhang, Y. F., “Analysis of deformation error of a novel fracture-resetting robot based on elastic deformation and vector superposition principle". Science Technology and Engineering, 19(35), 2019, pp.234-240.

3. Feng, Y. B., Zhao, X. H., He, Z., Li, Z. G., Wang, Y. B., "Depth hover control of spherical robot for internal detection of oilimmersed transformer". Control and Decision, 35(2), 2020, pp.375381.

4. Huynh, B. P., Wu, C. W., Kuo, Y. L., "Force/position hybrid control for a hexa robot using gradient descent iterative learning control algorithm". IEEE Access, (7), 2019, pp.72329-72342.

5. Zhang, W. Z., Dong, X. K., "Switched fuzzy-PD control of contact forces in robotic micromanipulation of drosophila larvae". In: Proceedings of the 2015 international conference on Robotics and Automation of electronic governance, New York, USA: IEEE, 64(5), 2017, pp.1169-1177.

6. Huang, X. R., Song, Y. Y., Li, Q. S., Xiao, X., "A vibration suppression algorithm for industrial robot joint servo system based on internal model control". Transactions of China Electrotechnical Society, 34(3), 2019, pp.497-505.

7. Liu, L. Y., "Design and application of internal model control for jointed cloud robot with delay time". Journal of Shijiazhuang University of Applied Technology, 31(4), 2019, pp.8-12.

8. Meng, Z. J., Xu, X. D., Cai, J., Huang, P. F., "Master-slave bilateral adaptive internal model control for space teleoperation robot". Computer Measurement and Control, 19(10), 2011, pp.2424-2426, 2429.

9. Luc, L. T., Alin, Albu, S., "Robust adaptive tracking control based on state feedback controller with integrator terms for elastic joint robots with uncertain parameters". IEEE Transactions on Control Systems Technology, 26(6), 2018, pp.2259-2267.

10. Baek, J., Cho, S., Han, S., "Practical time-delay control with adaptive gains for trajectory tracking of robot manipulators". IEEE Transactions on Industrial Electronics, 65(7), 2018, pp.5682-5692.

11. Rubio, Jesus, J. D., "Sliding mode control of robotic arms with deadzone". Institution of Engineering and Technology Control Theory and Applications, 11(8), 2017, pp.1214-1221.
12. Baek, J., Jin, M. L., Han S., "A new adaptive sliding-mode control scheme for application to robot manipulators". IEEE Transactions on Industrial Electronics, 63(6), 2016, pp.3628-3637.

13. Nair, R., Ravindranathan, N., Karki, H. K., Shukla, A., Behera, L. B., Jamshidi. M., "Fault-tolerant formation control of nonholonomic robots using fast adaptive gain nonsingular terminal sliding mode control”. IEEE Systems Journal, 13(1), 2019, pp.1006-1017.

14. Worthmann, K., Mehrez, M. W., Zanon, M., Mann, G. K. I., Gosine, R. G., Diehl, M., "Model predictive control of nonholonomic mobile robots without stabilizing constraints and costs”. IEEE Transactions on Control Systems Technology, 24(4), 2016, pp.1394-1406.

15. Chen, Y., Li, Z. J., Kong, H. Y., Fan, K., "Model predictive tracking control of nonholonomic mobile robots with coupled input constraints and unknown dynamics". IEEE Transactions on Industrial Informatics, 15(6), 2019, pp.3196-3205.

16. Incremona, G. P., Ferrara, A., Magni, L., "MPC for robot manipulators with integral sliding modes generation". IEEE/ASME Transactions on Mechatronics, 22(3), 2017, pp.1299-1307.

17. Liu, M. Z., Zhou, F. Y., Li, M., Wang, Y. G., Chen, K., "The composite control of backstepping control based on uncertain model compensation of wheeled mobile robot". Journal of Shandong University (Engineering Science), 49(6), 2019, pp.36-44.

18. Peng, J. S., Qiu, W. C., Li, J. F., Li, T. C., Song, L. Y., "Inversion adaptive sliding mode path tracking control for agricultural wheeled mobile robot". Computer Applications and Software, 36(11), 2019, pp.86-90.

19. Capolei, M. C., Andersen, N. A., Lund, H. H., Falotico, E., Tolu, S., "A cerebellar internal models control architecture for online sensorimotor adaptation of a humanoid robot acting in a dynamic environment". IEEE Robotics and Automation Letters, 5(1), 2020, pp.80-87.

20. Blagouchine, I. V., Moreau, E., "Control of a speech robot via an optimum neural network based internal model with constraints". IEEE Transactions on Robotics, 26(1), 2010, pp.142-159.

21. Yang, J., Shao, R., Xu, C. K., Chang, C. P., "Robust output tracking of flexible joint manipulator based on iterative learning". Journal of Harbin Engineering University, 39(1), 2018, pp. 106-113. 